\title{
Review on the Progress of Plant Immune Polysaccharides
}

\author{
Xuye Liu ${ }^{1, a}$, Ping Zhao ${ }^{1, b}$, Songyi Lin $^{1, c^{*}}$ \\ ${ }^{1}$ Jilin University College of Food Science and Engineering, Changchun, 130062, China

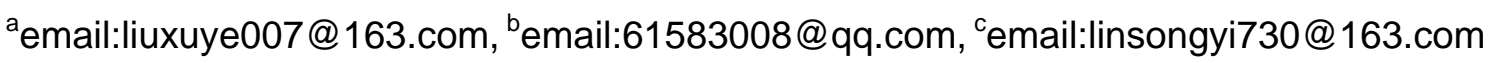

Keywords: Review, Progress, Plant Polysaccharides, Immunity

\begin{abstract}
Plant bioactive polysaccharides and the products of organisms are the hot focus of present research. They play various biological roles, one of the most vital of which is enhancing the immunity. Relationship between immune substances and disease has been confirmed through lots of studies. The immune ability of polysaccharides attributes to their stimulation of macrophage phagocytosis, activation of T-cells and B-cells. This paper reviews bioactive polysaccharides from plant sources focusing on their immune ability. In addition, extraction methods, structure characterization and activity evaluation are reviewed.
\end{abstract}

\section{Introduction}

In recent years, the study of polysaccharides and its complex substance have already been major topics. The structure detection methods and bioactivities also have been developed by the specialists. A large number of facts revealed that saccharide is a sort of informational molecule in organism, which participate in the reactions of physiological and pathological procedures, such as immune regulations, intercellular recognitions, intercellular material transport, etc[1]. As a matter of fact, polysaccharide is a kind of bioactive macromolecule substance, composed by many monosaccharides, exactly ten or more than ten,linked with glycoside bonds[2].

Nowadays, polysaccharides have been found widespread in animals, plants and fungus as the living matter[3]. More and more functions of polysaccharides have been discovered, thus this paper attempt to summarize the extraction methods and the features of polysaccharides.

\section{Extraction Methods}

Many different extraction methods have been used, in order to obtain polysaccharides from materials as much as possible, generally including the methods seen in Table 1.

Ultrasonic-assisted extraction technique was used in many fields, especially in the bioactive compose extraction. It can greatly improve the extraction efficiency, save solvent, avoid the effects of temperature on the extraction composition [4]. Kui Zhong [5] and Yong-guang Bi [5] adopted this method to extract Longan and Koidz polysaccharides with the yield of $15.13 \%$ which showed a greater advantage compared with other methods. Another superior extraction method is ultrasound-assisted enzymatic extraction, which is considered as a mild, efficient and environmental friendly extraction method. It has been used in the extraction of various kinds of compounds recently. The enzymes added to the solution like celluloses and proteases can promote the release of the bioactive contents through breaking the cell wall. In this way, Huaguo Chen [6] and Yong Liu [7] extracted Lycium barbarum polysaccharides and Astragalus polysaccharides, respectively, getting the yield of $6.31 \pm 0.03 \%$ and $29.96 \pm 0.14 \%$. In addition, many other conventional extraction procedures such as water [8-9], acid and alkali were found to be used in treating various materials. However, it showed that the weaker functions on the yield compared with enzyme and ultrasound-assisted extraction.

\section{Immunity Evaluations}

Plant polysaccharides have comprehensive bioactivities, anti-tumor, anti-cancer, hypoglycemic, liver protection and so on, especially in enhancing immunity of organisms [10]. Vivo experiments 
indicated that Lycium barbarum polysaccharides inhibit the growth of transplantable sarcoma S180 and increase macrophage phagocytosis, the form of antibody secreted by spleen cells, spleen lymphocyte proliferation, CTL activity, IL-2 mRNA expression level and reduce the lipid peroxidation in S180-bearing mice [11]. Ganoderma lucidum polysaccharides showed significant impacts on reducing the levels of serum IL-6 and TNF-alevels and increasing the levels of serum IL-2, IL-4 and IL-10 in GLP-treated mice compared to gastric cancer model mice [9-13], in addition, activating [12] and stimulating [13] macrophage proliferation. Koidz polysaccharides enhance both serum IgG response and gut mucosal immunity [14] and Fructus Jujubae significantly stimulated NO production in RAW264.7 macrophages, and promoted LPS-induced splenocyte proliferation [15]. Polysaccharides from King oyster mushroom [16] and Longan [17] not only inhibiting the tumor growth, but also increasing relative thymus and spleen indices. Vitro experiments showed that Astragalus polysaccharides regulate T-cell-mediated immunity via CD11chighCD45RBlow DCs [18]. In brief, these founding suggest that plant polysaccharides directly or indirectly enhance immunity through vivo and vitro means.

Table 1 Extraction methods and activity evaluation of some plant polysaccharides

\begin{tabular}{|c|c|c|c|c|}
\hline Materials & $\begin{array}{l}\text { Extraction } \\
\text { methods }\end{array}$ & $\begin{array}{c}\text { Extraction } \\
\text { rate }\end{array}$ & Activity evaluation & Reference \\
\hline Koidz & Ultrasonic & $15.13 \%$ & Vivo, enhance serum IgG response & [4] \\
\hline Astragalus & Enzyme & $\begin{array}{c}29.96 \pm \\
0.14 \%\end{array}$ & Vitro, regulate T-cell-mediated immunity & {$[5,6]$} \\
\hline $\begin{array}{l}\text { Lycium } \\
\text { barbarum }\end{array}$ & $\begin{array}{l}\text { Ultrasound-assisted } \\
\text { enzymatic }\end{array}$ & $\begin{array}{l}6.31 \pm \\
0.03 \%\end{array}$ & $\begin{array}{l}\text { Vivo, as vaccine enhancement of } \\
\text { humoral immunity by activating Tfh } \\
\text { cells }\end{array}$ & {$[7,8,9]$} \\
\hline $\begin{array}{l}\text { Fructus } \\
\text { Jujubae }\end{array}$ & Water & $\begin{array}{l}6.47 \pm \\
0.26 \%\end{array}$ & $\begin{array}{l}\text { Vivo, test RAW264.7 macrophages, } \\
\text { splenocyte }\end{array}$ & {$[10]$} \\
\hline King oyster & Water & $9.36 \%$ & Vivo, inhibite the tumor growth & {$[11][12]$} \\
\hline $\begin{array}{l}\text { Ganoderma } \\
\text { lucidum }\end{array}$ & Water & $1.45 \%$ & $\begin{array}{l}\text { Vivo, improve the cancer mice, reduce } \\
\text { levels of IL-1b, IL-6 and TNF-a in } \\
\text { mice,GLP stimulation to the macrophage } \\
\text { proliferation and higher nitric oxide } \\
\text { production }\end{array}$ & {$[13,14]$} \\
\hline Longan & Ultrasonic & $\begin{array}{c}\text { Not } \\
\text { mentioned }\end{array}$ & $\begin{array}{l}\text { Vivo, potent immune - modulatory } \\
\text { tumor mice }\end{array}$ & {$[15][16]$} \\
\hline
\end{tabular}

\section{Structure characterizations}

In recent years, more and more attentions are attached to the study of plant polysaccharide structure. It is not difficult to image that polysaccharides from various organisms have significant disparities, their molecular weight via from $2.1 \times 10^{4}$ Da to $5.47233 \times 10^{6}$ Da [12]. The structure features of plant polysaccharides are normally investigated by some or all of the techniques including FT-IR, GC-MS, NMR, SEM, Smith degradation [13]. Previous analysis indicated that Lycium barbarum polysaccharide was composed with rhamnose, arabinose, xylose, galactose, mannose, galacturonic acid of $1.00: 7.85: 0.37: 0.65: 3.01: 8.16$ with average molecular weight of $2.25 \times 10^{6}$ Da [14]. Astragalus polysaccharide was composed with glucose, and a small amount of arabinose and xylose, with their molar ratios of $0.54: 1: 18.14$ and $0.23: 1: 29.39$ [18]. Other plant polysaccharide structures have listed in Table2. 
Table 2 Composition, characterization and molecular of some plant polysaccharides

\begin{tabular}{|c|c|c|c|c|}
\hline Material & Composition & Analysis method & $\begin{array}{c}\text { Molecular } \\
\text { weight }\end{array}$ & Reference \\
\hline Aloe vera & $\begin{array}{l}\text { Man: Glc: Gal: GalA: Fuc: Ara: Xy } \\
\text { l= 120:9:6:3:2:2:1 }\end{array}$ & $\begin{array}{l}\text { GC-MS, }{ }^{1} \mathrm{H}-\mathrm{NMR} \\
\text { and }{ }^{13} \mathrm{C}-\mathrm{NMR}\end{array}$ & $\begin{array}{l}\text { Not } \\
\text { mentioned }\end{array}$ & {$[12]$} \\
\hline Green tea & Galactose, $\beta$-(1,4)-linked & $\begin{array}{l}\text { IR,GC,Smith } \\
\text { degradation, NMR }\end{array}$ & $\begin{array}{l}2.1247 \times \\
10^{4} \mathrm{Da}\end{array}$ & [13] \\
\hline Longan & Glu: Man: Ara= 14.16:6.40:1.00 & $\begin{array}{l}\text { GC-MS, HPLC, } \\
\text { SEC,FTIR,NMR }\end{array}$ & $\begin{array}{c}5.47233 \times \\
10^{6} \mathrm{Da}\end{array}$ & {$[14]$} \\
\hline $\begin{array}{l}\text { Lycium } \\
\text { barbarum }\end{array}$ & $\begin{array}{l}\text { Rha, Ara, xylose, Gla, Man, Glaacid } \\
=1.00: 7.85: 0.37: 0.65: 3.01: 8.16\end{array}$ & $\begin{array}{l}\text { FT-IR,GC-MS, } \\
\text { NMR }\end{array}$ & $\begin{array}{c}2.25 \times 10^{6} \\
\mathrm{Da}\end{array}$ & {$[16]$} \\
\hline Astragalus & Glu: Ara :xylose= 0.54:1:18.14 & $\begin{array}{c}\text { FT-IR, Smith } \\
\text { degradation, NMR }\end{array}$ & $\begin{array}{l}4.77 \times \\
10^{6} \mathrm{Da}\end{array}$ & {$[17]$} \\
\hline $\begin{array}{l}\text { Ganoderma } \\
\text { lucidum }\end{array}$ & Glu, Gla, Man, Ara & GC-MS,NMR & $\begin{array}{c}6.1 \times 10^{4} \\
\text { to } 18.4 \times \\
10^{4} \mathrm{Da}\end{array}$ & [18] \\
\hline $\begin{array}{l}\text { Fructus } \\
\text { Jujubae }\end{array}$ & RQP1d and RQP2d & FT-IR,SEM & $\begin{array}{c}2.068 \times \\
10^{5} \mathrm{Da} \\
\end{array}$ & {$[18]$} \\
\hline
\end{tabular}

\section{Conclusion}

With the developing researches of polysaccharides deeply and comprehensively, more and more new types of polysaccharides are found, from the structure analysis to the bioactivities. Today, functional polysaccharides have been reported to have a range effects, including anticancer, antitumor anticoagulation, antivirus, enhancing human immunity. Polysaccharides are found to be responsible for these bioactivities. However, the future challenge is to get the better understanding of the structure-bioactivity relationship and more insight in the structural features. It is also a good opportunity for the scientists to elucidate the biological roles of polysaccharides and design high potential antitumor drugs based on the high order structures. The application and the future of polysaccharides in the functional food will be extensive and glorious.

\section{Acknowledgement}

The authors acknowledge the financial support provided by the Changchun Science and Technology Plan Program (201317NK03) and the Youth Scientific Innovation Leading Talent and Team Building Project of Jilin Province (20140519014JH).

\section{References}

[1] Yongguang Bi, Dinglong Yang, Xiaojun Huang, Yumin Li, Minxia Huang. Study on Ultrasonic-assisted extraction of polysaccharide of atractylis macroceohala Koidz of experiment [J]. energy procedia, 201217 1778-1785.

[2] Zhong Kui, Qiang Wang, Yin He, Xuanhui He. Evaluation of radicals scavenging, immunity-modulatory and antitumor activities of longan polysaccharides with ultrasonic extraction on in S180 tumor mice models [J]. International Journal of Biological Macromolecules, 201047 (3) 356-360.

[3] Huaguo Chen, Xin Zhou, Junzeng Zhang. Optimization of enzyme assisted extraction of polysaccharides from astragalus membranaceus [J]. Carbohydrate Polymers, 2014111 (20) 567-575.

[4] Yong Liu, Guili Gong, Jiao Zhang, Shaoyi Jia, Feng Li, Yanyan Wang, Songhai Wu. Response surface optimization of ultrasound-assisted enzymatic extraction polysaccharides from Lycium 
barbarum [J]. Carbohydrate Polymers, 2014110 (38) 278-284.

[5] Guoting Cui, Wuxia Zhang, Qingjie Wang, Amin Zhang, Haibo Mu, Hongjin Bai, Jinyou Duan. Extraction Optimization, characterization and immunity activity of polysaccharides from Fructus Jujubae [J]. Carbohydrate Polymers, 2014111 (20) 245-255.

[6] Ke Pan, Qunguang Jiang, Guoqing Liu, Xiongying Miao, Dewu Zhong. Optimization extraction of ganoderma Lucidum polysaccharides and its immunity and antioxidant activities [J]. International Journal of Biological Macromolecules, 201355 (2) 301-306.

[7] Xinkui Liu, , Lin Wang, Chunmei Zhang, Hongmin Wang, Xiaohong Zhang, Yuexia Li. Structure characterization and antitumor activity of a polysaccharide from the Alkaline extract of king oyster mushroom [J]. Carbohydrate Polymers, 2015118 101-106.

[8] Shenshen Zhang, Shaoping Nie, Danfei Huang, Wenjuan Li, Mingyong Xie. Immunomodulatory effect of ganoderma atrum polysaccharide on CT26 tumor-bearing mice [J]. Food Chemistry, 2013136 (3-4) 1213-1219.

[9] Lu Gan, Shenghua Zhang, Xiangliang Yang, Huibi Xu. Immunomodulation and antitumor activity by a polysaccharide-protein complex from Lycium barbarum [J]. International Immunopharmacology, 20044 (4) 563-569.

[10] Xiaoping Chen, Yana Chen, Shuibing Li, Youguo Chen, Jianyun Lan, Lanping Liu. Free radical scavenging of ganoderma Lucidum polysaccharides and its effect on antioxidant enzymes and immunity activities in cervical carcinoma rats [J]. Carbohydrate Polymers, 200977 (2) 389-393.

[11] Min Shi, Zhenya Zhang, Yingnan Yang. Antioxidant and immunoregulatory activity of ganoderma Lucidum polysaccharide (GLP) [J]. Carbohydrate Polymers, 201395 (1) 200-206.

[12] Yuanfeng Wang, Xinlin Wei, Zhengyu Jin. Structure analysis of a neutral polysaccharide isolated from green tea [J]. Food Research International, 200942 (5-6) 739-745.

[13] Zhong Kui, Qiang Wang, Yin He, Xuanhui He. Evaluation of radicals scavenging, immunity-modulatory and antitumor activities of Longan polysaccharides with ultrasonic extraction on in S180 tumor mice models [J]. International Journal of Biological Macromolecules, 201047 (3) 356-360.

[14] Qingyang Liu, Yongming Yao, Shuwen Zhang, Zhiyong Sheng. Astragalus polysaccharides regulate $\mathrm{T}$ Cell-mediated immunity via CD11cHighCD45RBlow DCs in vitro [J]. Journal of Ethnopharmacology, 2011136 (3) 457-64.

[15] Xiaoping Chen, Yan Chen, Shuibing Li, Youguo Chen, Jianyun Lan, Lanping Liu. Free radical scavenging of Ganoderma lucidum polysaccharides and its effect on antioxidant enzymes and immunity activities in cervical carcinoma rats [J]. Carbohydrate Polymers, 200977 (2) 389-393.

[16] Jianguo Wang, Zhaocheng Ma, Lina Zhang, Yapeng Fang, Fatang Jiang, Glyn O. Phillips. Structure and chain conformation of water-soluble heteropolysaccharides from Ganoderma lucidum [J]. Carbohydrate Polymers, 20112 (2) 844-851.

[17] Chunxia Su, Xiangguo Duan, Lijun Liang, Feng Wang, Jie Zheng, Xueyan Fu, Yamei Yang, Ling Huang, Ningping Wang. Lycium barbarum polysaccharides as an adjuvant for recombinant vaccine through enhancement of humoral immunity by activating Tfh cells [J]. Veterinary Immunology and Immunopathology, 2013158 (1-2) 98-104.

[18] Longyun Hao, Rui Wang, Li Zhang, Kuanjun Fang, Yajing Men, Zongzhong Qi, Peng Jiao, Jianwei Tian, Jingquan Liu. Utilizing cellulase as a hydrogen peroxide stabilizer to combine the biopolishing and bleaching procedures of cotton cellulose in one bath [J]. Cellulose, 201321 (1) $1-13$. 